\title{
Anodic Oxidation of Ethylene and Acetylene
}

\section{THE ELECTROCATALYTIC ROLE OF GOLD ELECTRODES}

\author{
C. Cwiklinski and J. Perichon \\ Laboratoire d'Electrochimie Organique de l'Université Paris, Val de Marne, France
}

Most research on the electro-oxidation of aqueous solutions of ethylene and acetylene, with the exception of work on the synthesis of ethylene glycol from ethylene and of acetic acid from acetylene, has been directed towards the use of these hydrocarbons in fuel cells.

Ethylene and acetylene can be electro-oxidised at noble metal electrodes. These can be divided into two groups: the first, including platinum, rhodium and iridium, which give total oxidation of the hydrocarbons to carbon dioxide gas; the second, including gold and palladium, gives partial oxidation and carbon dioxide gas is rarely the main product (1-8).

This work is concerned with identifying the intermediate products formed during the electrooxidation of ethylene and acetylene at gold electrodes, and studying the variables affecting their formation. The effects of these variables ( $\mathrm{pH}$ of the solution, hydrocarbon concentration, temperature, rotation speed of the electrode) were studied from currentpotential curves at rotating gold anodes. The data thus obtained were used to define the experimental conditions required for electrolysis at controlled potentials. Finally, possible mechanisms for the oxidation of ethylene and acetylene at gold anodes are formulated.

\section{Electrochemical Oxidation of Aqueous Solutions of Ethylene}

Study of the electrochemical behaviour of aqueous solutions of ethylene at rotating gold micro-electrodes from current-potential curves $(9,10)$ led to the following conclusions:

(a) The electro-oxidation is not diffusion controlled but results from prior adsorption at the electrodes.

(b) Ethylene is only electro-active in acid media $(\mathrm{pH}<4.8)$. Under these conditions its electrooxidation is not $\mathrm{pH}$ dependent and takes place in two stages: the first occurs in a potential area ranging from 0.8 to $0.97 \mathrm{~V} / \mathrm{NHE}$, and the second from $1.12 \mathrm{~V} / \mathrm{NHE}$ up to the gold oxidation potential at the $\mathrm{pH}$ considered.

(c) The first oxidation step definitely involves a single electron.
Identical results are obtained at large gold electrodes. These electrodes (gauzes $10 \times 5 \mathrm{~cm}, 196$ mesh $\left(\mathrm{cm}^{2}\right)$ were of the type used as consumption electrodes in potentiostatic electrolysis.

These assumptions suggest that the nature and distribution of the products formed during electrolysis of aqueous solutions of ethylene depend on the potential applied to the gold anode, the acidity of the solution, the temperature and the electricity input.

In general the products formed in these controlled potential electrolysis at $0.95 \mathrm{~V} / \mathrm{NHE}$ and $1.2 \mathrm{~V} / \mathrm{NHE}$ are ethanol, formol, glyoxol, acetic acid, glycol aldehyde, carbon dioxide gas, as well as traces of other oxygenated products: $\mathrm{HCOOH},(\mathrm{COOH})_{2}$, $\left(\mathrm{CH}_{3}\right)_{2} \mathrm{CO}(10,11)$.

Although the products formed at the two operating potentials were identical, their distribution was found to be very different. Thus at $25^{\circ} \mathrm{C}$, and for a similar electrical input the faradaic yield of partial oxidation products was found to be higher at $1.2 \mathrm{~V} / \mathrm{NHE}$ than at $0.95 \mathrm{~V} / \mathrm{NHE}$. In addition, it seems that at a potential of $0.95 \mathrm{~V} / \mathrm{NHE}$, the partial oxidation products form at the beginning of the reaction and are then probably transformed into $\mathrm{CO}_{2}$, while at $1.2 \mathrm{~V} / \mathrm{NHE}$ this reaction is much slower. Thus, at this potential, the plot of the quantity of partial oxidation products formed versus the amount of electricity supplied gives a straight line. An increase in temperature was also observed to favour the formation of partial oxidation products at both potentials. Finally, at $80^{\circ} \mathrm{C}$ and $0.95 \mathrm{~V} / \mathrm{NHE}$ ethanol was observed to be the main product formed, subsequently followed by acetic acid so that

$$
\frac{\mathrm{CH}_{3} \mathrm{CHO}}{\mathrm{CH}_{3} \mathrm{CO}_{2} \mathrm{H}} \geqslant 1
$$

while at $1.2 \mathrm{~V} / \mathrm{NHE}$ the partial oxidation products form competitively with acetic acid rapidly becoming the main product. Similar experiments conducted in other acidic aqueous media (10) give identical results.

The addition of non-electroactive ethanol in acidic medium at the start of electrolysis at $0.95 \mathrm{~V} /$ NHE, tends to inhibit the ethylene oxidation reaction to partial oxidation products. This effect is hardly noticeable at $1.2 \mathrm{~V} / \mathrm{NHE}$. 
Thus, the potential applied to the gold electrode constitutes an essential factor in the formation of the various partial oxidation products of ethylene, and gold plays a predominant electrocatalytic role in the oxidation process.

The results obtained support an anodic oxidation mechanism for ethylene initially identical to those reported by other workers $(1,3,4,12)$. This would involve the adsorption of an active chemical species with the same degree of oxidation as acetaldehyde such as $\left(\mathrm{CH}_{3} \mathrm{CHOH}\right)^{+}$adsorbed. Adsorption of this species being stronger at $0.95 \mathrm{~V} / \mathrm{NHE}$, thus leading to a more complete oxidation while at $1.2 \mathrm{~V}$ adsorption being weaker, the intermediate species would compete between desorption and complete oxidation.

\section{Electrical Oxidation of Aqueous Solutions of Acetylene}

Few papers describe the anodic oxidation of aqueous solutions of acetylene at gold electrodes (5, 13), especially with regard to the nature and quantity of products formed during the electrolysis of these solutions. Our work is mainly concerned with this study.

The results obtained from current-potential curves at rotating gold micro-electrodes show that acetylene is oxidised at all pHs by a mechanism involving the exchange of one electron and one proton. As for ethylene, the electro-oxidation process is governed by adsorption, and from $0.85 \mathrm{~V} / \mathrm{NHE}$ a solid deposit is formed at the surface of the electrode (14).

Electrolysis was carried out under the same experimental conditions as for ethylene, i.e. $0.95 \mathrm{~V} /$ $\mathrm{NHE}$ and $1.2 \mathrm{~V} / \mathrm{NHE}$ respectively, in acid media. On applying the potential, the oxidation current density was observed to increase initially, and eventually to stabilise. Simultaneously the electrode became brown or brownish black, while the electrolyte became yellowish.

Acetic acid, glyoxol, glycodaldehyde, formaldehyde, and carbon dioxide gas were identified and measured in the electrolyte. The presence of acetaldehyde, a hydration product of acetylene was also observed (this forming only when the potential is applied). At ambient temperature, in an $\mathrm{N} \mathrm{H}_{2} \mathrm{SO}_{4}$ medium, the faradaic yield $\rho$ for partial oxidation products is approximately 40 per cent (15), whatever the coulombic input: i.e. $10<p<20$ for $\mathrm{CH}_{3} \mathrm{COOH}, 5<p<15$ for $\mathrm{CHOCHO}, 5<p<10$ for $\mathrm{CH}_{2} \mathrm{OHCHO}$ and approximately 5 per cent for $\mathrm{HCHO}$. These results have been observed both at $0.95 \mathrm{~V} / \mathrm{NHE}$ and $1.2 \mathrm{~V} /$ NHE, which confirms the hypothesis for a simple electro-oxidation mechanism.

These results are somewhat different from those published by other authors $(5,13)$. In particular we have not been able to confirm the formation of a polymer (13) on the electrode. Our analyses have led to the conclusion that the deposit on the electrode largely consists of gold, gold salts and/or gold oxides $(14,15)$.

Formation of this deposit, which occurs even at low potentials such as $0.95 \mathrm{~V} / \mathrm{NHE}$ could be explained by the formation of a gold and acetylene complex which would facilitate gold oxidation. Therefore, there is an obvious interaction between gold and acetylene at potentials between $0.95 \mathrm{~V} / \mathrm{NHE}$ and 1.2V/NHE, while for ethylene only hydrocarbon oxidation occurred. Thus, the mechanism for the oxidation of acetylene in aqueous acid solutions would involve corrosion of the gold anode. The formation of acetaldehyde during electrolysis could also be related to a catalytic phenomenon going through a complex $\left(\mathrm{Au}-\mathrm{C}_{2} \mathrm{H}_{2}\right)$.

\section{Conclusion}

This work has demonstrated that the electrolytic oxidation of ethylene and acetylene at gold anodes can be controlled, even at ambient temperatures.

For ethylene, gold plays an important electrocatalytic role probably linked to electro-adsorption of intermediate species; this would explain the formation of a complex mixture of products, the distribution of which is potential-dependent.

For acetylene, however, the electrocatalytic role is more complex, as the nature of the electrode surface seems to change from polished gold to gold oxides, salts and/or organic complexes, this being independent of the oxidation potential considered.

\section{References}

1 H. Dahms and J. O'M. Bockris, f. Electrochem. Soc., 1964, 111, (6), 728

2 A. T. Kuhn, H. Wroblowa and J. O'M. Bockris, Trans. Faraday Soc., 1967, 63, (6), 1458

3 J. W. Johnson, S. C. Lai and W. J. James, Electrochim. Acta, 1970, 15, (9), 1511

4 S. C. Lai, Diss. Abstr. B, 1969, 30, (4), 1660

5 T. N. Hartley and D. Price, F. Electrochem. Soc., 1970, 117, (4), 448

6 F. Goodridge, Chem. Process Engng., 1966, 47, (3), 121

7 A. R. Blake, J. G. Sunderland and A. T. Kuhn, $\mathcal{F}$. Chem. Soc. $(A), 1969,(19), 3015$

8 F. Goodridge and C. J. H. King, Trans. Faraday Soc., 1970, 66, (575), 2889

9 C. Cwiklinski and J. Perichon, C.r., Sér. C, 1969, 269, (24), 1480

10 C. Cwiklinski and J. Perichon, Electrochim. Acta, 1974, 19, (6) 297

11 C. Cwiklinksi and J. Perichon, C.r., Sér. C, 1971, 272, (24), 1930

$12 \mathrm{~J}$. O'M. Bockris and H. Wroblowa, F. Electroanal. Chem., $1964,7,(6), 428$

13 J. W. Johnson, J. L. Reed and W. J. James, f. Electrochem. Soc., 1967, 114, (6), 572

14 C. Cwiklinski, Thèse de Doctorat d'Etat és Sciences Physique Université Paris VI (1972-C.N.R.S. AO 7149)

15 C. Cwiklinski and J. Perichon, Electrochim. Acta, 1974, 19, (3), 315 